\title{
THE GLOBAL STABILITY ANALYSIS FOR AN SIS MODEL WITH AGE AND INFECTION AGE STRUCTURES
}

M-1551

February 2001

\section{Yicang Zhou \\ Baojun Song \\ Zhien Ma}

Keywords: age-structure, infection age, proportionate mixing, basic reproductive number, global stability.

Abstract: A general SIS model with chronological age and infection age structures is formulated. We analyze the global dynamics of the model with a constructive iteration procedure. The basic reproductive number $R_{0}$ is calculated using the next generation operator approach. $\mathbf{R}_{0}$ plays a sharp threshold role in determining the global dynamics, i.e., the endemic steady-state is globally asymptotically stable if $\mathrm{R}_{0}>1$, while the disease-free steady state is globally asymptotically stable if $R_{0} \geq$ 1. the basic reproductive number is over estimated where the infection age is ignored. 


\title{
The Global Stability Analysis for an SIS Model with Age and Infection Age Structures
}

\author{
Yicang Zhou ${ }^{1}$ Baojun Song ${ }^{2}$ Zhien $\mathrm{Ma}^{1}$ \\ ${ }^{1}$ Department of Mathematics, Xi'an Jiaotong University, Xi'an, 710049, China \\ ${ }^{2}$ Department of Biometrics, Cornell University, Ithaca, NY 14853-7801, USA
}

\begin{abstract}
A general SIS model with chronological age and infection age structures is formulated. We analyze the global dynamics of the model with a constructive iteration procedure. The basic reproductive number $R_{0}$ is calculated using the next generation operator approach. $R_{0}$ plays a sharp threshold role in determining the global dynamics, i.e., the endemic steady-state is globally asymptotically stable if $R_{0}>1$, while the disease-free steady-state is globally asymptotically stable if $R_{0} \leq 1$. The basic reproductive number is over estimated where the infection age is ignored.
\end{abstract}

Keywords: age-structure, infection age, proportionate mixing, basic reproductive number, global stability.

\section{Introduction}

Virtually all epidemic models for communicable diseases in homogeneous populations have been inspired by the model derived by Kermack and Mckendrick [1,2]. Epidemiologically, this model, in its general form only became well-known in the 1970s[3,4,5]. Chronological and infection age might be the most important factors in disease spread. Vynnycky and Fine [6], for instance, have shown that tuberculosis(TB) infection is at low rate for individuals less than 10 years old, but dramatically increases when the individual's age is between 10 and 20 years. The mixing structure of a population is often closely related to age structure of the population. Contact

\footnotetext{
*Author to whom correspondence should be addressed. E-mail: zhouyc@mail.xjtu.edu.cn
} 
rates are highly dependent on age. Many diseases, for example, childhood diseases, may be mostly transmitted to individuals of the same age. Vaccination strategies for specific diseases are naturally applied to different age groups( see Castillo-Chavez and Feng[7] and references therein). Hence, during the last two decades of the 20th century, age-structured epidemic models have been extensively studied $[8,9,10,11,12,13,14,15,16]$. Dietz and Schenzle studied an epidemic model with special forms of age and infectious age dependent contact rate[17]. Thieme and Castillo-Chavez formulated a model and explored the role of variable infectivity in combination with a variable incubation period in the dynamics of HIV transmission in homogeneously mixing population[18]. Proportionate mixing has been extensively used in age-structure models. This mixing structure has been useful in the study of dynamics of childhood diseases. The use of proportionate mixing also makes it easier to get an explicit formula for the basic reproductive number[19].

Previous dynamical analysis for many age-structure models has been incomplete. The local stability for disease-free steady-state is easy to establish for most age-structured models when the basic reproductive number is less then a unity. The globally asymptotic stability of a stable age distribution, however, is very difficult in general. In this paper, we focus on the study of the global dynamics of two-age structured model. The study is theoretical in nature, but the framework and the approach may be applicable to specific diseases.

The paper is organized as follows: Section 2 introduces the epidemic model. The basic reproductive number is computed and fundamental assumptions are spelled out. Section 3 establishes the global asymptotic stability of the disease-free steady-state and the endemic non-uniform age-distributions. An iteration procedure is used. The appendix shows that the solutions of the epidemic model are nonnegative for relevant initial distributions.

\section{Model and basic reproductive number}

The framework of our model is from Busenberg and Castillo-Chavez [20]. SIS models with both chronological age and infection age structures are formulated. Demographically the population is stratified by chronological age and, epidemiologically, it is partitioned into susceptible and infective. Here $s(a, t)$ denotes the density of susceptibles at time $t$ and $i(a, c, t)$ the density of infectives at time $t$, where $a$ is the chronological age and $c$ is the infection age, that is, the time 
span since infection. The variable $c$ does not distinguish individuals and can never be greater than the chronological age $a$. The total numbers of the susceptibles $S(t)$ and infectives $I(t)$ at time $t$ are given by $S(t)=\int_{0}^{A} s(a, t) d a, I(t)=\int_{0}^{A} \int_{0}^{a} i(a, c, t) d c d a$, respectively, where $A$ is the maximum age. The total population size is given by $P(t)=S(t)+I(t)=\int_{0}^{A} s(a, t) d a+$ $\int_{0}^{A} \int_{0}^{a} i(a, c, t) d c d a$. It is assumed that all newborns are susceptibles and the disease is not fatal. Thus, we ignore the disease-related mortality. The model describing the dynamics of an SIS infectious disease takes the form of a nonlinear hyperbolic system of PDEs:

$$
\begin{aligned}
& \frac{\partial s}{\partial a}+\frac{\partial s}{\partial t}=-\mu(a) s(a, t)-G(a, t)+\gamma(a) \int_{0}^{a} i(a, c, t) d c, \\
& s(0, t)=\int_{A_{1}}^{A_{2}} b(a, P(t)) p(a, t) d a \\
& s(a, 0)=s_{0}(a), \quad s(A, t)=0 \\
& \frac{\partial i}{\partial a}+\frac{\partial i}{\partial c}+\frac{\partial i}{\partial t}=-(\mu(a)+\gamma(a)) i(a, c, t), \\
& i(a, 0, t)=G(a, t)=C(a) s(a, t) \int_{0}^{A} \int_{0}^{a^{\prime}} \beta\left(a^{\prime}, c\right) \frac{i\left(a^{\prime}, c, t\right)}{p\left(a^{\prime}, t\right)} \rho\left(a, a^{\prime}, t\right) d c d a^{\prime}, \\
& i(A, c, t)=0 \\
& i(a, c, 0)=i_{0}(a, c)
\end{aligned}
$$

where $p(a, t)=s(a, t)+\int_{0}^{a} i(a, c, t) d c$ is the entire population density at time $t ; P(t)=$ $\int_{0}^{A} p(a, t) d a$ the total population size at time $t ; b(a, P(t))$ the density-dependent age-specific birth rate(birth modulus) of the population; $s_{0}(a)$ and $i_{0}(a, c)$ are the initial distributions; $\left[A_{1}, A_{2}\right]$ is the fecundity period, $0<A_{1}<A_{2}<A ; \mu(a)$ the age-specific mortality rate; $\gamma(a)$ the age-specific recovery rate of the infective individuals; $C(a)$ the age-specific contact rate; $\beta(a, c)$ the age-specific probability that a susceptible becomes infected given that it had a contact with an infectious of $(a, c)$ type; $\rho\left(a, a^{\prime}, t\right)$ the probability that an individual of age $a$ has contact with an individual of $a^{\prime}$ given that it has a contact. We implicitly assume that the population mixes proportionately [20], that is, $\rho\left(a, a^{\prime}, t\right)=\rho\left(a^{\prime}, t\right)=\frac{C\left(a^{\prime}\right) p\left(a^{\prime}, t\right)}{\int_{0}^{A} C(a) p(a, t) d a}$, The force of infection(incidence), that is, the rate at which susceptibles individuals of age $a$ move over into the infective class per capita and per unit of time, is given by $G(a, t)$;

In the most cases (except for the case where backward bifurcation takes place) the basic reproductive number regulates the local dynamics of disease transmission., i.e., if it is less than a unity, the disease dies out, while if it is greater than one the disease establishes itself. The main 
result of this paper is the establishment of the global nature of this transcritical bifurcation. The next generation operator is used to find out the basic reproductive number. This approach formulated by Diekmann et al. [19] has been widely used recently. Feng, Castillo-Chavez, and Huang have a detailed version of this method and specific applications in this volume. First, we compute the demographic steady-state and expected infectivity. The demographic steady-state is calculated from Equation (1a) by letting $i\left(a, a^{\prime}, t\right)=0$ ( details are provided later in this section). It turns out that the demographic steady-state

$$
p_{\infty}(a)=P_{\infty} \frac{\exp \left(-\int_{0}^{a} \mu(\tau) d \tau\right)}{\int_{0}^{A} \exp \left(-\int_{0}^{a} \mu(\tau) d \tau\right) d a}, \quad \text { where } P_{\infty} \text { is a constant. }
$$

Let $E\left(a, a^{\prime}, c\right)$ denote the expected infectivity of an infectious individual of $\left(a^{\prime}, c\right)$ type towards a susceptible of age $a$ at the demographic steady-state. $E\left(a, a^{\prime}, c\right)$ is determined by a routine infection process thought that follows the pattern of an infective who first survives from $a^{\prime}-c$ to $a^{\prime}$; has contacts with susceptibles; and transmits the disease by chance. It is assumed that these events happen independently. Hence, $E\left(a, a^{\prime}, c\right)$ is the product of three terms: the survival probability of an infected individual surviving from $a^{\prime}-c$ to $a^{\prime}$; the contact rate of individuals of age $a$ with individuals of age $a^{\prime}$; and the transmission probability. Therefore

$$
E\left(a, a^{\prime}-c, c\right)=C(a) \rho\left(a^{\prime}\right) \beta\left(a^{\prime}, c\right) \frac{\pi\left(a^{\prime}, c\right)}{p_{\infty}\left(a^{\prime}\right)},
$$

where $\rho\left(a^{\prime}\right)=\frac{C\left(a^{\prime}\right) p_{\infty}\left(a^{\prime}\right)}{\int_{0}^{A} C(a) p_{\infty}(a) d a}$ and $\pi\left(a^{\prime}, c\right)=e^{-\int_{a^{\prime}-c}^{a^{\prime}}(\mu(a)+\gamma(a)) d a}($ the survival probability of an infected individual survives from $a^{\prime}-c$ to $a^{\prime}$ ).

Hence

$$
\begin{aligned}
R_{0} & =\int_{0}^{A} \int_{0}^{a^{\prime}} C\left(a^{\prime}-c\right) p_{\infty}\left(a^{\prime}-c\right) \rho\left(a^{\prime}\right) \beta\left(a^{\prime}, c\right) \frac{\pi\left(a^{\prime}, c\right)}{p_{\infty}\left(a^{\prime}\right)} d c d a^{\prime} \\
& =\int_{0}^{A} \int_{0}^{a^{\prime}} C\left(a^{\prime}-c\right) p_{\infty}\left(a^{\prime}-c\right) \frac{C\left(a^{\prime}\right) \beta\left(a^{\prime}, c\right)}{\int_{0}^{A} C\left(a^{\prime}\right) p_{\infty}\left(a^{\prime}\right) d a^{\prime}} \pi\left(a^{\prime}, c\right) d c d a^{\prime}
\end{aligned}
$$

\section{Dynamics analysis}

\subsection{Assumptions}

From the epidemiological and mathematical point of view, we make the following assumptions:

- A1. $\mu(a)>\mu_{0}>0$ is a positive continuous function on $[0, A)$, and $\int_{0}^{A} \mu(a) d a=+\infty$. Set $M(a)=\exp \left(-\int_{0}^{a} \mu(\tau) d \tau\right)$ for $0 \leq a<A$, and $M(A)=0$. It is obvious that $M(a)$ is a 
continuous, decreasing function on $[0, A]$, and $0 \leq M(a) \leq 1$ for $0 \leq a \leq A$.

- A2. $b(a, P)$ is a nonnegative continuous function, and $b(a, P)>0$ for $A_{1}<a<A_{2}$, $b(a, P)=0$ for $a \leq A_{1}$ or $a \geq A_{2}$.

- A3. $p_{0}(a) \geq 0$ is a continuous function, $p_{0}(a)>0$ if $a \in[0, A)$, and $p_{0}(A)=0$. The initial data $p_{0}(a)$ satisfies the compatible condition

$$
p_{0}(0)=\int_{0}^{A} b\left(a, \int_{0}^{A} p_{0}(\tau) d \tau\right) p_{0}(a) d a
$$

which is simply the requirement that the initial data be consistent with the birth process.

- A4. $\gamma(a)$ is a nonnegative and continuous function on $[0, A]$.

- A5. The effects of infection age follows an exponential distribution with an expected infection age $\frac{1}{\delta}$ and $\beta\left(a^{\prime}, c\right)$ takes a form of variable-separated, $\beta\left(a^{\prime}, c\right)=\beta_{1}\left(a^{\prime}\right) e^{-\delta c}$, where $\beta_{1}\left(a^{\prime}\right)$ is assumed to be bounded, positive and continuous. To keep notation simpler, we set $\lambda_{1}(a)=\frac{C(a)}{\int_{0}^{A} C\left(a^{\prime}\right) p_{\infty}\left(a^{\prime}\right) d a^{\prime}}, \lambda_{2}\left(a^{\prime}\right)=\beta_{1}\left(a^{\prime}\right) C\left(a^{\prime}\right), \lambda\left(a, a^{\prime}, c\right)=\lambda_{1}(a) \lambda_{2}\left(a^{\prime}\right) e^{-\delta c}$, and $\lambda^{*}=\max _{0 \leq a \leq A, 0 \leq a^{\prime} \leq A} \lambda\left(a, a^{\prime}, c\right)$

- A6. $i_{0}(a, c)$ is bounded, nonnegative and continuous function on $0 \leq c \leq a \leq A$. And $i_{0}(a, c)$ satisfies the continuous compatible condition

$$
\left(p_{0}(a)-\int_{0}^{a} i_{0}(a, c) d c\right) \int_{0}^{A} \int_{0}^{a^{\prime}} \lambda\left(a, a^{\prime}, c\right) i_{0}\left(a^{\prime}, c\right) d c d a^{\prime}=i_{0}(a, 0) .
$$

The lack of disease-induced mortality in our model implies that the total population density $p(a, t)$ is governed by the following demographic evolution equation $[21,22]$ :

$$
\begin{aligned}
& \frac{\partial p}{\partial a}+\frac{\partial p}{\partial t}=-\mu(a) p(a, t) \\
& p(0, t)=\int_{A_{1}}^{A_{2}} b(a, P(t)) p(a, t) d a \\
& p(a, 0)=p_{0}(a), \quad p(A, t)=0
\end{aligned}
$$

where $p_{0}(a)=s_{0}(a)+\int_{0}^{a} i_{0}(a, c) d c$. Under assumptions $(\mathrm{A} 1) \sim(\mathrm{A} 3)$, the age-structured population model (4) is well-posed [23,24]. From the net reproductive number $n(P)=\int_{0}^{A} b(a, P) M(a) d a$, we see that, if the equation $n(P)=1$ has a positive root $P_{\infty}$, then the total population density $p(a, t)$ has a steady-state $p_{\infty}(a)=P_{\infty} M(a) / \int_{0}^{A} M(a) d a[23]$. Sufficient conditions for the local 
stability for the positive steady-state $p_{\infty}(a)$ can be found in $[25,26,27]$. We focus on the global stability of non-uniform epidemic steady-state throughout the rest of this paper.

We assume that the total population is at its demographic steady-state $p_{\infty}(a)$ i.e., $p(a, t)=$ $p_{\infty}(a)=P_{\infty} M(a) / \int_{0}^{A} M(a) d a$, thus replacing $s(a, t)$ by $p_{\infty}(a)-\int_{0}^{a} i(a, c, t) d c$ in the force of infection, $G(a, t)$, we arrive at a single equation in terms of $i(a, c, t)$.

$$
\begin{aligned}
& \frac{\partial i}{\partial a}+\frac{\partial i}{\partial c}+\frac{\partial i}{\partial t}=-(\mu(a)+\gamma(a)) i(a, c, t), \\
& i(a, 0, t)=G(a, t)=\left(p_{\infty}(a)-\int_{0}^{a} i(a, c, t) d c\right) \int_{0}^{A} \int_{0}^{a^{\prime}} \lambda\left(a, a^{\prime}, c\right) i\left(a^{\prime}, c, t\right) d c d a^{\prime}, \\
& i(A, c, t)=0 \\
& i(a, c, 0)=i_{0}(a, c) .
\end{aligned}
$$

Using a similar idea by Tucker et al., it can be shown that the Equation (5) has a unique continuous solution for all $t \geq 0$, provided that the assumptions (A1) (A6) hold[28]. Are the solutions to the Equation (5) nonnegative? Theorem A below gives a positive answer.

Theorem 1 Assume that $(A 1) \sim(A 6)$ hold. Let $i(a, c, t)$ be the solution of (5) with the initial distribution $i_{0}(a, c)$ satisfying $\int_{0}^{a} i_{0}(a, c) d c \leq p_{\infty}(a)$. Then $i(a, c, t) \geq 0$ for all $t \geq 0$.

The proof of Theorem 1 can be found in Appendix.

\subsection{Global stability of disease-free steady-state}

Equation (5) can be explicitly solved along the characteristic lines,

$$
\begin{aligned}
& \quad i(a, c, t)=G(a-c, t-c) \pi(a, c) \\
& \text { where } \quad G(a-c, t-c)=i_{0}(a-t, c-t) \frac{\pi(a, t)}{\pi(a, c)}, \text { if } t \leq c .
\end{aligned}
$$

Imposing the boundary condition (5b) upon (6) gives the following nonlinear equation for $G(a, t)$

$$
\begin{aligned}
G(a, t) & =\left(p_{\infty}(a)-\int_{0}^{a} G(a-c, t-c) \pi(a, c) d c\right) \\
& \times \int_{0}^{A} \int_{0}^{a^{\prime}} \lambda\left(a, a^{\prime}, c\right) G\left(a^{\prime}-c, t-c\right) \pi\left(a^{\prime}, c\right) d c d a^{\prime} .
\end{aligned}
$$

Since $\pi\left(a^{\prime}, c\right)=\exp \left(-\int_{a^{\prime}-c}^{a^{\prime}}(\mu(\tau)+\gamma(\tau)) d \tau\right)$ is bounded, positive, and continuous, it follows from (6) that the asymptotic behavior of $i(a, c, t)$ is completely determined by the asymptotic 
behavior of $G(a, t)$, which satisfies Equation (7). Therefore, Equation (7) is the main target in our analysis.

The specific expression for the basic reproductive number for Model (5) under the assumptions $(\mathrm{A} 1) \sim(\mathrm{A} 6)$ is

$$
R_{0}=\int_{0}^{A} \int_{0}^{a^{\prime}} \lambda_{1}\left(a^{\prime}-c\right) \lambda_{2}\left(a^{\prime}\right) e^{-\delta c} p_{\infty}\left(a^{\prime}-c\right) \pi\left(a^{\prime}, c\right) d c d a^{\prime} .
$$

To see the effect of infection age, we look at $R_{0}$ as a function of the expected infection age, $\frac{1}{\delta}$. $R_{0}\left(\frac{1}{\delta}\right)$ increases as does $\frac{1}{\delta}$. An extreme case $\frac{1}{\delta}=\infty$ corresponds to the situation where the infection age has no effect. $R_{0}\left(\frac{1}{\delta}\right)$ approaches its maximum value $R_{0}=\int_{0}^{A} \int_{0}^{a^{\prime}} \lambda_{1}\left(a^{\prime}-\right.$ c) $\lambda_{2}\left(a^{\prime}\right) p_{\infty}\left(a^{\prime}-c\right) \pi\left(a^{\prime}, c\right) d c d a^{\prime}$ that is the corresponding basic reproductive number to the case where only chronological age is considered. Hence the basic reproductive number in single age-structure model over estimates the severity of the epidemic.

We now examine the stability of the disease-free steady-state and the existence of an endemic steady-state.

Theorem 2 Assume that $(A 1) \sim(A 6)$ hold. Then the disease-free steady-state is globally asymptotically stable if $R_{0} \leq 1$, whereas, it is unstable and there exists a unique endemic steady-state if $R_{0}>1$.

Proof. The local stability of the disease-free steady-state is directly derived from the definition of $R_{0}$. What remain now is to show that it is a global attractor. Defining

$$
\begin{gathered}
\lambda_{3}\left(a^{\prime}, c\right)=\lambda_{2}\left(a^{\prime}\right) e^{-\delta c}, \\
w(t)=\int_{0}^{A} \int_{0}^{a^{\prime}} \lambda_{3}\left(a^{\prime}, c\right) i\left(a^{\prime}, c, t\right) d c d a^{\prime},
\end{gathered}
$$

one can see that

$$
w(t)=\int_{0}^{A} \int_{0}^{a^{\prime}} \lambda_{3}\left(a^{\prime}, c\right) G\left(a^{\prime}-c, t-c\right) \pi\left(a^{\prime}, c\right) d c d a^{\prime}, \quad t \geq A .
$$

According to Equation (7), we arrive at

$$
\begin{aligned}
& w(t)=\int_{0}^{A} \int_{0}^{a^{\prime}} \lambda_{1}\left(a^{\prime}-c\right) \lambda_{3}\left(a^{\prime}, c\right) p_{\infty}\left(a^{\prime}-c\right) \pi\left(a^{\prime}, c\right) w(t-c) d c d a^{\prime} \\
& -\int_{0}^{A} \int_{0}^{a^{\prime}} \lambda_{1}\left(a^{\prime}-c\right) \lambda_{3}\left(a^{\prime}, c\right) \int_{0}^{a^{\prime}-c} G\left(a^{\prime}-c-\tau, t-c-\tau\right) \pi\left(a^{\prime}, c-\tau\right) w(t-c) d \tau d c d a^{\prime},
\end{aligned}
$$


and

$$
w(t) \leq \int_{0}^{A} \int_{0}^{a^{\prime}} \lambda_{1}\left(a^{\prime}-c\right) \lambda_{3}\left(a^{\prime}, c\right) p_{\infty}\left(a^{\prime}-c\right) \pi\left(a^{\prime}, c\right) w(t-c) d c d a^{\prime} .
$$

Let $\left\|p_{\infty}(a)\right\|$ and $\left\|i_{0}(a, c)\right\|$ be the maximum norm of the continuous functions, that is, $\left\|p_{\infty}(a)\right\|=$ $\max _{0 \leq a \leq A} p_{\infty}(a),\left\|i_{0}(a, c)\right\|=\max _{0 \leq c \leq a \leq A} i_{0}(a, c)$. The assumption (A6) and the expression of $p_{\infty}(a)=P_{\infty} M(a) / \int_{0}^{A} M(a) d a$ imply that $\left\|p_{\infty}(a)\right\|$ and $\left\|i_{0}(a, c)\right\|$ are well-defined positive numbers. The fact that the disease-free steady-state $i(a, c, t)=0$ is a trivial solution to (5) and the regularity result of solutions implies that $i(a, c, t)$, the solutions of (5) with the initial distribution $i_{0}(a, c)$, are continuously dependent on $i_{0}(a, c)$. Hence, for any given positive number $\varepsilon$, there exists a positive number $\delta$, such that $i(a, c, t)<\varepsilon$ if $\left\|i_{0}(a, c)\right\|<\delta$ and $t \leq A$. From (5b) it follows that $0 \leq G(a, t) \leq c_{1} \varepsilon$ for $0 \leq t \leq A$, here $c_{1}$ is a positive constant. From the definition of $w(t)$, there also exists a positive constant $c_{0}$, such that $w(t)<c_{0} \varepsilon$ for $0 \leq t \leq A$.

If $R_{0}<1$, Inequality (10) implies that $w(A) \leq R_{0} c_{0} \varepsilon<\frac{R_{0}+1}{2} c_{0} \varepsilon<c_{0} \varepsilon$. We claim that $w(t)<\frac{R_{0}+1}{2} c_{0} \varepsilon$ for all $A \leq t \leq 2 A$. Otherwise, there exists at least one $t_{0} \in(A, 2 A]$, such that $w\left(t_{0}\right)=\frac{R_{0}+1}{2} c_{0} \varepsilon$, and $w(t)<\frac{R_{0}+1}{2} c_{0} \varepsilon$ for all $A \leq t<t_{0}$. From Inequality (10) it follows that

$$
\begin{aligned}
w\left(t_{0}\right) & \leq \int_{0}^{A} \int_{0}^{a^{\prime}} \lambda_{1}\left(a^{\prime}-c\right) \lambda_{3}\left(a^{\prime}, c\right) p_{\infty}\left(a^{\prime}-c\right) \pi\left(a^{\prime}, c\right) w\left(t_{0}-c\right) d c d a^{\prime} \\
& \leq R_{0} c_{0} \varepsilon<\frac{R_{0}+1}{2} c_{0} \varepsilon .
\end{aligned}
$$

This contradiction implies that $w(t)<\frac{R_{0}+1}{2} c_{0} \varepsilon$ for all $A \leq t \leq 2 A$. Mathematical induction gives

$$
w(t) \leq\left(\frac{R_{0}+1}{2}\right)^{n} c_{0} \varepsilon \text { for } n A \leq t \leq(n+1) A .
$$

From (5b) and (11) we obtain

$$
G(a, t) \leq\left(\frac{R_{0}+1}{2}\right)^{n} c_{2} \varepsilon, \text { for } n A \leq t \leq(n+1) A .
$$

Finally the expression (6) gives rise to

$$
i(a, c, t) \leq\left(\frac{R_{0}+1}{2}\right)^{n} c_{3} \varepsilon, \text { for } n A \leq t \leq(n+1) A .
$$

$c_{2}$ and $c_{3}$ in (12) and (13) are positive constants. Inequality (13) says that the disease-free steadystate $i(a, c, t)=0$ is a globally asymptotically attractive. Thus it is globally asymptotically stable since we have known it is local stable. 
To prove the existence of unique stable age distribution, we need to look for the timeindependent solution $G_{\infty}(a)$ of the equation (7) when $R_{0}>1 . G_{\infty}(a)$ satisfies the equation

$$
\begin{array}{cc} 
& G_{\infty}(a)=\lambda_{1}(a)\left(p_{\infty}(a)-\int_{0}^{a} G_{\infty}(\tau) \frac{N(a)}{N(\tau)} d \tau\right) w^{*} \\
\text { where } & w^{*}=\int_{0}^{A} \int_{0}^{a^{\prime}} \lambda_{3}\left(a^{\prime}, c\right) G_{\infty}\left(a^{\prime}-c\right) \pi\left(a^{\prime}, c\right) d c d a^{\prime} \\
& N(a)=\exp \left(-\int_{0}^{a}(\mu(\tau)+\gamma(\tau)) d \tau\right) .
\end{array}
$$

Defining $g(a)=\frac{G_{\infty}(a)}{\lambda_{1}(a)}$, it can be verified that $g(a)$ is the solution of the equation

$$
\begin{gathered}
g(a)=\left(p_{\infty}(a)-\int_{0}^{a} g(\tau) \lambda_{1}(\tau) \frac{N(a)}{N(\tau)} d \tau\right) w^{*} \\
\text { where } \quad w^{*}=\int_{0}^{A} \int_{0}^{a^{\prime}} \lambda_{3}\left(a^{\prime}, c\right) g\left(a^{\prime}-c\right) \lambda_{1}\left(a^{\prime}-c\right) \pi\left(a^{\prime}, c\right) d c d a^{\prime}
\end{gathered}
$$

Changing the integral equation for $g(a)$ into a differential equation, solving the resulting equation, a closed form for $g(a)$ is obtained,

$$
g(a)=w^{*} N(a) \exp \left(-w^{*} \int_{0}^{a} \lambda_{1}(\tau) d \tau\right)\left(p_{\infty}(0)+\int_{0}^{a} \frac{\gamma(\tau) p_{\infty}(\tau) \exp \left(w^{*} \int_{0}^{\tau} \lambda_{1}(\theta) d \theta\right)}{N(\tau)} d \tau\right)
$$

The substitution of (16) into (15b) leads to that (16) is the solution of (15) if and only if $w^{*}$ is the solution of the equation $f\left(w^{*}\right)=1$, where

$$
\begin{aligned}
& f\left(w^{*}\right)=\int_{0}^{A} \int_{0}^{a^{\prime}} \lambda_{1}\left(a^{\prime}-c\right) \lambda_{3}\left(a^{\prime}, c\right) N\left(a^{\prime}\right) \exp \left(-w^{*} \int_{0}^{a^{\prime}-c} \lambda_{1}(\tau) d \tau\right) \\
& \times\left(p_{\infty}(0)+\int_{0}^{a^{\prime}-c} \frac{\gamma(\tau) p_{\infty}(\tau) \exp \left(w^{*} \int_{0}^{\tau} \lambda_{1}(\theta) d \theta\right)}{N(\tau)} d \tau\right) d c d a^{\prime} \\
& =p_{\infty}(0) \int_{0}^{A} \int_{0}^{a^{\prime}} \lambda_{1}\left(a^{\prime}-c\right) \lambda_{3}\left(a^{\prime}, c\right) N\left(a^{\prime}\right) \exp \left(-w^{*} \int_{0}^{a^{\prime}-c} \lambda_{1}(\tau) d \tau\right) d c d a^{\prime} \\
& +p_{\infty}(0) \int_{0}^{A} \int_{0}^{a^{\prime}} \lambda_{1}\left(a^{\prime}-c\right) \lambda_{3}\left(a^{\prime}, c\right) N\left(a^{\prime}\right) \int_{0}^{a^{\prime}-c} \gamma(\tau) \exp \left(-w^{*} \int_{\tau}^{a^{\prime}-\tau} \lambda_{1}(\theta) d \theta+\int_{0}^{\tau} \gamma(\theta) d \theta\right) d \tau d c d a^{\prime} .
\end{aligned}
$$

$f\left(w^{*}\right)$ is a monotonic decreasing function of $w^{*}$, with $\lim _{w^{*} \rightarrow+\infty} f\left(w^{*}\right)=0$, and

$$
\begin{aligned}
f(0) & \left.=\int_{0}^{A} \int_{0}^{a^{\prime}} \lambda_{1}\left(a^{\prime}-c\right) \lambda_{3}\left(a^{\prime}, c\right) p_{\infty}(0)\left(1+\int_{0}^{a^{\prime}-c} \gamma(\tau) \exp \left(\int_{0}^{\tau} \gamma(\theta) d \theta\right) d \tau\right)\right) N\left(a^{\prime}\right) d c d a^{\prime} \\
& =\int_{0}^{A} \int_{0}^{a^{\prime}} \lambda_{1}\left(a^{\prime}-c\right) \lambda_{3}\left(a^{\prime}, c\right) p_{\infty}(0) \exp \left(\int_{0}^{a^{\prime}-c} \gamma(\tau) d \tau\right) N\left(a^{\prime}\right) d c d a^{\prime} \\
& =\int_{0}^{A} \int_{0}^{a^{\prime}} \lambda_{1}\left(a^{\prime}-c\right) \lambda_{3}\left(a^{\prime}, c\right) p_{\infty}\left(a^{\prime}-c\right) \pi\left(a^{\prime}, c\right) d c d a^{\prime}=R_{0}>1
\end{aligned}
$$


This follows that there exists one and only one solution to equation $f\left(w^{*}\right)=1$ by using the intermediate theorem for continuous function. Hence the existence of a unique steady age distribution is established.

Now turn to prove that the disease-free steady-state is unstable when $R_{0}>1$. If it is stable then for the positive number $\varepsilon=\frac{R_{0}-1}{2 A^{3} \lambda^{*}}$, there exists a $\delta>0$, such that, $0 \leq i_{0}(a, c)<\delta$ implies that

$$
0 \leq i(a, c, t)<\varepsilon, \quad 0 \leq c \leq a \leq A, \quad t \geq 0 .
$$

By the definition of $w(t)$ and (17), we know

$$
0<w(t)=\int_{0}^{A} \int_{0}^{a^{\prime}} \lambda_{3}\left(a^{\prime}, c\right) i\left(a^{\prime}, c, t\right) d c d a^{\prime} \leq A^{2} \lambda^{*} \varepsilon, \quad t \geq 0 .
$$

On the other hand, if $t \geq A,(6)$ and (9) yield

$$
\begin{aligned}
w(t) & =\int_{0}^{A} \int_{0}^{a^{\prime}} \lambda_{3}\left(a^{\prime}, c\right)\left(p_{\infty}\left(a^{\prime}-c\right)-\int_{0}^{a^{\prime}-c} i\left(a^{\prime}-c, \tau, t-c\right) d \tau\right) w(t-c) \pi\left(a^{\prime}, c\right) d c d a^{\prime} \\
& \geq \int_{0}^{A} \int_{0}^{a^{\prime}} \lambda_{3}\left(a^{\prime}, c\right)\left(p_{\infty}\left(a^{\prime}-c\right)-\int_{0}^{a^{\prime}-c} i\left(a^{\prime}-c, \tau, t-c\right) d \tau\right) \pi\left(a^{\prime}, c\right) d c d a^{\prime} \min _{t-A \leq \tau \leq t} w(\tau) \\
& \geq\left(R_{0}-A^{3} \lambda^{*} \varepsilon\right) \min _{t-A \leq \tau \leq t} w(\tau)=\frac{R_{0}+1}{2} \min _{t-A \leq \tau \leq t} w(\tau),
\end{aligned}
$$

from which we can have

$$
w(t) \geq\left(\frac{R_{0}+1}{2}\right)^{n} \min _{0 \leq \tau \leq A} w(\tau), \quad n A \leq t \leq(n+1) A
$$

The initial distribution $i_{0}(a, c)$ can be chosen such that $\min _{0 \leq \tau \leq A} w(\tau)=w^{0}>0$. Consequently, Inequality (18) and (20) can not be true simultaneously. This contradiction implies the instability of the disease free equilibrium. The proof of Theorem 2 is complete.

The threshold result (Theorem 2) presents a satisfactory answer to the stability of the diseasefree steady-state and the existence and uniqueness of nontrivial stead-state. It is natural to investigate the stability of the unique endemic steady-state when $R_{0}>1$.

\subsection{The global stability of the endemic steady-state}

The two-age-structured system is replaced by two coupled single-age-structured system. A comparison theorem then is established for the coupled models. Finally, we prove the global stability results for the SIS model (2). 
We first define the age and weighted age density functions

$$
j(a, t)=\int_{0}^{a} i(a, c, t) d c, \quad k(a, t)=\int_{0}^{a} e^{-\delta c} i(a, c, t) d c,
$$

which are governed by

$$
\begin{aligned}
& \frac{\partial j}{\partial a}+\frac{\partial j}{\partial t}=-(\mu(a)+\gamma(a)) j+\left(p_{\infty}(a)-j(a, t)\right) \lambda_{1}(a) \int_{0}^{A} \lambda_{2}\left(a^{\prime}\right) k\left(a^{\prime}, t\right) d a^{\prime} \\
& \frac{\partial k}{\partial a}+\frac{\partial k}{\partial t}=-(\mu(a)+\gamma(a)+\delta) k+\left(p_{\infty}(a)-j(a, t)\right) \lambda_{1}(a) \int_{0}^{A} \lambda_{2}\left(a^{\prime}\right) k\left(a^{\prime}, t\right) d a^{\prime} \\
& j(a, 0)=j_{0}(a)=\int_{0}^{a} i_{0}(a, c) d c \\
& k(a, 0)=k_{0}(a)=\int_{0}^{a} e^{-\delta c} i_{0}(a, c) d c \\
& j(0, t)=0, \quad j(A, t)=0 \\
& k(0, t)=0, \quad k(A, t)=0 .
\end{aligned}
$$

Going one step further, we perform the normalization,

$$
u(a, t)=e^{-\delta a} j(a, t) / p_{\infty}(a), \quad v(a, t)=k(a, t) / p_{\infty}(a)
$$

thus

$$
0 \leq u(a, t) \leq v(a, t) \leq 1, \quad 0 \leq e^{\delta a} u(a, t) \leq 1
$$

A new system in terms $u(a, t)$ and $v(a, t)$ is

$$
\begin{gathered}
\frac{\partial u}{\partial a}+\frac{\partial u}{\partial t}=F(u, v)(a, t), \\
\frac{\partial v}{\partial a}+\frac{\partial v}{\partial t}=G(u, v)(a, t), \\
u(a, 0)=u_{0}(a)=e^{-\delta a} j_{0}(a) / p_{\infty}(a), \\
v(a, 0)=v_{0}(a)=k_{0}(a) / p_{\infty}(a), \\
u(0, t)=0, \quad v(0, t)=0, \\
\text { where } \quad F(u, v)(a, t)=-(\gamma(a)+\delta) u(a, t)+\left(e^{-\delta a}-u(a, t)\right) \lambda_{1}(a) \int_{0}^{A} \lambda_{2}\left(a^{\prime}\right) p_{\infty}\left(a^{\prime}\right) v\left(a^{\prime}, t\right) d a^{\prime}, \\
G(u, v)(a, t)=-(\gamma(a)+\delta) v(a, t)+\left(1-e^{\delta a} u(a, t)\right) \lambda_{1}(a) \int_{0}^{A} \lambda_{2}\left(a^{\prime}\right) p_{\infty}\left(a^{\prime}\right) v\left(a^{\prime}, t\right) d a^{\prime} .
\end{gathered}
$$

When $R_{0}>1$, it is straightforward to show that the unique endemic steady-state of (2) 
corresponds to the unique endemic steady-state of $(22)$ :

$$
\begin{aligned}
& u_{\infty}(a)=\int_{0}^{a} V e^{-\delta \tau} \lambda_{1}(\tau) \exp \left(-\int_{\tau}^{a}\left(\gamma(\theta)+\delta+V \lambda_{1}(\theta)\right) d \theta\right) d \tau \\
& v_{\infty}(a)=\int_{0}^{a} V\left(1-e^{\delta \tau} u_{\infty}(\tau)\right) \lambda_{1}(\tau) \exp \left(-\int_{\tau}^{a}(\gamma(\theta)+\delta) d \theta\right) d \tau
\end{aligned}
$$

where

$$
V=\int_{0}^{A} \lambda_{2}(a) p_{\infty}(a) v_{\infty}(a) d a
$$

A comparison theorem for the constructed System of PDEs (22) is established below:

Theorem 3 (Comparison Theorem) Assume that (A1) (A6) hold. Let $u_{1}(a, t), u_{2}(a, t)$, $v_{1}(a, t), v_{2}(a, t)$ be the solutions of (22) with the initial conditions $u_{1}(a, 0)=u_{10}(a), u_{2}(a, 0)=$ $u_{20}(a), v_{1}(a, t)=v_{10}(a), v_{2}(a, 0)=v_{20}(a)$, respectively. Then (1) $u_{1}(a, t) \leq u_{2}(a, t), v_{1}(a, t) \leq$ $v_{2}(a, t)$ if $0 \leq u_{20}(a)-u_{10}(a) \leq v_{20}(a)-v_{10}(a)$. (2) $\xi u_{1}(a, t) \leq u_{\xi}(a, t)$ and $\xi v_{1}(a, t) \leq v_{\xi}(a, t)$, where $u_{\xi}(a, t)$ and $v_{\xi}(a, t)$ are the solutions of (22) with the initial conditions $u_{\xi}(a, 0)=\xi u_{10}(a)$, $v_{\xi}(a, 0)=\xi v_{10}(a)$, and $\xi$ is a constant $0 \leq \xi<1$.

Proof. Let $\eta$ be a positive constant such that

$$
\eta \max _{0 \leq a \leq A}\left(\gamma(a)+2 \delta+\lambda_{1}(a) \exp (2 \delta A) \int_{0}^{A} \lambda_{2}(a) p_{\infty}(a) d a\right)<1 .
$$

The characteristics method implies that (22) is equivalent to the following system of integral equations

$$
\begin{array}{rlrl}
u(a, t) & =\exp \left(\frac{-t}{\eta}\right) u_{0}(a-t)+\frac{1}{\eta} \int_{0}^{t} \exp \left(\frac{-(t-\tau)}{\eta}\right)(u(a-t+\tau, \tau) \\
& +\eta F(u, v)(a-t+\tau, \tau)) d \tau, & & a \geq t, \\
u(a, t) & =\frac{1}{\eta} \int_{0}^{a} \exp \left(\frac{-(a-\tau)}{\eta}\right)(u(\tau, t-a+\tau) & \\
& +\eta F(u, v)(\tau, t-a+\tau)) d \tau, & & a<t, \\
v(a, t) & =\exp \left(\frac{-t}{\eta}\right) v_{0}(a-t)+\frac{1}{\eta} \int_{0}^{t} \exp \left(\frac{-(t-\tau)}{\eta}\right)(v(a-t+\tau) \\
& +\eta G(u, v)(a-t+\tau, \tau)) d \tau, & & a \geq t, \\
v(a, t) & =\frac{1}{\eta} \int_{0}^{a} \exp \left(\frac{-(a-\tau)}{\eta}\right)(v(\tau, t-a+\tau) & \\
& +\eta G(u, v)(\tau, t-a+\tau)) d \tau, & & a<t .
\end{array}
$$


Construct two sequences by iteration

$$
\begin{aligned}
& u^{(0)}(a, t)=u_{0}(a), \\
& u^{(n+1)}(a, t)=\exp \left(\frac{-t}{\eta}\right) u_{0}(a-t)+\frac{1}{\eta} \int_{0}^{t} \exp \left(\frac{-(t-\tau)}{\eta}\right)\left(u^{(n)}(a-t+\tau, \tau)\right. \\
& \left.+\eta F\left(u^{(n)}, v^{(n)}\right)(a-t+\tau, \tau)\right) d \tau, \quad a \geq t, \\
& u^{(n+1)}(a, t)=\frac{1}{\eta} \int_{0}^{a} \exp \left(\frac{-(a-\tau)}{\eta}\right)\left(u^{(n)}(\tau, t-a+\tau)\right. \\
& \left.+\eta F\left(u^{(n)}, v^{(n)}\right)(\tau, t-a+\tau)\right) d \tau, \quad a<t . \\
& v^{(0)}(a, t)=v_{0}(a) \\
& v^{(n+1)}(a, t)=\exp \left(\frac{-t}{\eta}\right) v_{0}(a-t)+\frac{1}{\eta} \int_{0}^{t} \exp \left(\frac{-(t-\tau)}{\eta}\right)\left(v^{(n)}(a-t+\tau, \tau)\right. \\
& \left.+\eta G\left(u^{(n)}, v^{(n)}\right)(a-t+\tau, \tau)\right) d \tau, \quad a \geq t \\
& v^{(n+1)}(a, t)=\frac{1}{\eta} \int_{0}^{a} \exp \left(\frac{-(a-\tau)}{\eta}\right)\left(v^{(n)}(\tau, t-a+\tau)\right. \\
& \left.+\eta G\left(u^{(n)}, v^{(n)}\right)(\tau, t-a+\tau)\right) d \tau, \quad a<t,
\end{aligned}
$$

It is not difficult to show that $u^{(n)}(a, t)$ and $v^{(n)}(a, t)$ converge uniformly to $u(a, t)$ and $v(a, t)$, the solutions of the integral equations (22). If $0 \leq u^{(0)}(a, t) \leq v^{(0)}(a, t) \leq 1,0 \leq e^{\delta a} u^{0}(a, t) \leq 1$, $0 \leq u^{(n)}(a, t) \leq v^{(n)}(a, t) \leq 1$, and $0 \leq e^{\delta a} u^{(n)}(a, t) \leq 1$, then from (24b) and the selection for 
$\eta$, we have that

$$
\begin{aligned}
0 & \leq u^{(n+1)}(a, t) \\
& =\exp \left(\frac{-t}{\eta}\right) u_{0}(a-t)+\frac{1}{\eta} \int_{0}^{t} \exp \left(\frac{-(t-\tau)}{\eta}\right)\left(u^{(n)}(a-t+\tau, \tau)\right. \\
& -\eta e^{-\delta(a-t+\tau)}(\gamma(a-t+\tau)+\delta) \\
& +\eta\left(e^{-\delta(a-t+\tau)}-u^{(n)}(a-t+\tau, \tau)\right)((\gamma(a-t+\tau)+\delta) \\
& \left.\left.+\lambda_{1}(a-t+\tau) \int_{0}^{A} \lambda_{2}\left(a^{\prime}\right) p_{\infty}\left(a^{\prime}\right) v^{(n)}\left(a^{\prime}, \tau\right) d a^{\prime}\right)\right) d \tau \\
& \leq \exp \left(\frac{-t}{\eta}-\delta a\right)+\frac{1}{\eta} \int_{0}^{t} \exp \left(\frac{-(t-\tau)}{\eta}\right)\left(u^{(n)}(a-t+\tau, \tau)\right. \\
& \left.-\eta e^{-\delta(a-t+\tau)}(\gamma(a-t+\tau)+\delta)+e^{-\delta(a-t+\tau)}-u^{(n)}(a-t+\tau, \tau)\right) d \tau \\
& \leq \exp \left(\frac{-t}{\eta}-\delta a\right)+\frac{1}{\eta} \int_{0}^{t} \exp \left(\frac{-(t-\tau)}{\eta}\right)(1-\eta \delta) e^{-\delta(a-t+\tau)} d \tau \\
& \leq \exp \left(\frac{-t}{\eta}-\delta a\right)+\left(1-\exp \left(\frac{-t}{\eta}+\delta t\right)\right) e^{-\delta a} \\
& \leq \exp \left(\frac{-t}{\eta}-\delta a\right)+\left(1-\exp \left(\frac{-t}{\eta}\right)\right) e^{-\delta a} \leq e^{-\delta a} \leq 1
\end{aligned}
$$

A similar procedure implies that

$$
0 \leq e^{\delta a} u^{(n+1)}(a, t) \leq 1, \quad 0 \leq v^{(n+1)}(a, t) \leq 1
$$

Based on (24), a relationship between both sequences is deduced

$$
\begin{aligned}
& u^{(n+1)}(a, t)-v^{(n+1)}(a, t)=\exp \left(\frac{-t}{\eta}\right)\left(u_{0}(a-t)-v_{0}(a-t)\right) \\
& +\frac{1}{\eta} \int_{0}^{t}\left(\operatorname { e x p } ( \frac { - ( t - \tau ) } { \eta } ) \left(( 1 - \eta ( \gamma ( a - t + \tau ) + \delta ) ) \left(u^{(n)}(a-t+\tau, \tau)\right.\right.\right. \\
& \left.-v^{(n)}(a-t+\tau, \tau)\right)+\eta\left(e^{-\delta(a-t+\tau)}-1\right)\left(1-e^{\delta(a-t+\tau)} u^{(n)}(a-t+\tau, \tau)\right) \\
& \left.\times \lambda_{1}(a-t+\tau) \int_{0}^{A} \lambda_{2}\left(a^{\prime}\right) p_{\infty}\left(a^{\prime}\right) v^{(n)}\left(a^{\prime}, \tau\right) d a^{\prime}\right) d \tau \leq 0, \quad a \geq t, \\
& u^{(n+1)}(a, t)-v^{(n+1)}(a, t) \\
& =\frac{1}{\eta} \int_{0}^{a}\left(\operatorname { e x p } ( \frac { - ( a - \tau ) } { \eta } ) \left(( 1 - \eta ( \gamma ( \tau ) + \delta ) ) \left(u^{(n)}(\tau, a-t+\tau)\right.\right.\right. \\
& \left.-v^{(n)}(\tau, a-t+\tau)\right)+\eta\left(e^{-\delta \tau}-1\right)\left(1-e^{\delta \tau} u^{(n)}(\tau, a-t+\tau)\right) \\
& \left.\times \lambda_{1}(\tau) \int_{0}^{A} \lambda_{2}\left(a^{\prime}\right) p_{\infty}\left(a^{\prime}\right) v^{(n)}\left(a^{\prime}, t-a+\tau\right) d a^{\prime}\right) d \tau \leq 0, \quad a \leq t,
\end{aligned}
$$

which implies $0 \leq u^{(n)}(a, t) \leq v^{(n)}(a, t) \leq 1$. It can be seen that $0 \leq e^{\delta a} u^{(n)}(a, t) \leq 1$ for all 
integer $n$ by induction. Hence,

$$
\begin{aligned}
& \lim _{n \rightarrow \infty} u^{(n)}(a, t)=u(a, t) \leq e^{-\delta a}, \\
& \lim _{n \rightarrow \infty} v^{(n)}(a, t)=v(a, t) \leq 1, \\
& 0 \leq u(a, t) \leq v(a, t) \leq 1, \\
& 0 \leq k(a, t) \leq j(a, t) \leq p_{\infty}(a), \quad 0 \leq a \leq A, \quad 0 \leq t \leq T .
\end{aligned}
$$

We claim that

$$
u_{1}^{(n)}(a, t) \leq u_{2}^{(n)}(a, t) \quad \text { and } \quad v_{1}^{(n)}(a, t) \leq v_{2}^{(n)}(a, t)
$$

hold for any integer $n$. Now we apply induction again to ensure the claim. Assume they are true for $n$, i.e.,

$$
\begin{aligned}
& 0 \leq u_{1}^{(n)}(a, t) \leq v_{1}^{(n)}(a, t) \leq 1, \quad 0 \leq u_{2}^{(n)}(a, t) \leq v_{2}^{(n)}(a, t) \leq 1 \\
\text { and } \quad & 0 \leq u_{2}^{(n)}(a, t)-u_{1}^{(n)}(a, t) \leq e^{\delta a}\left(v_{2}^{(n)}(a, t)-v_{1}^{(n)}(a, t)\right)
\end{aligned}
$$

Then a direct computation gives

$$
\begin{aligned}
& u_{2}^{(n+1)}(a, t)-u_{1}^{(n+1)}(a, t) \\
& =\exp \left(\frac{-t}{\eta}\right)\left(u_{2}^{(0)}(a, t)-u_{1}^{(0)}(a, t)\right) \\
& +\frac{1}{\eta} \int_{0}^{t} \exp \left(\frac{-(t-\tau)}{\eta}\right)\left(\left(u_{2}^{(n)}(a-t+\tau, \tau)-u_{1}^{(n)}(a-t+\tau, \tau)\right)\right. \\
& \times\left(1-\eta\left(\gamma(a-t+\tau)+\delta+\lambda_{1}(a-t+\tau) \int_{0}^{A} \lambda_{2}\left(a^{\prime}\right) p_{\infty}\left(a^{\prime}\right) v_{2}^{(n)}\left(a^{\prime}, \tau\right) d a^{\prime}\right)\right) \\
& +\eta e^{-\delta(a-t+\tau)} \lambda_{1}(a-t+\tau)\left(1-e^{\delta(a-t+\tau)} u_{1}^{(n)}(a-t+\tau, \tau)\right) \\
& \left.\times \int_{0}^{A} \lambda_{2}\left(a^{\prime}\right) p_{\infty}\left(a^{\prime}\right)\left(v_{2}^{(n)}\left(a^{\prime}, \tau\right)-v_{1}^{(n)}\left(a^{\prime}, \tau\right)\right) d a^{\prime}\right) d \tau \geq 0, \quad a \geq t,
\end{aligned}
$$

and

$$
\begin{aligned}
& u_{2}^{(n+1)}(a, t)-u_{1}^{(n+1)}(a, t) \\
& =\frac{1}{\eta} \int_{0}^{a} \exp \left(\frac{-(a-\tau)}{\eta}\right)\left(\left(u_{2}^{(n)}(\tau, t-a+\tau)-u_{1}^{(n)}(\tau, t-a+\tau)\right)\right. \\
& \times\left(1-\eta\left(\gamma(\tau)+\delta+\lambda_{1}(\tau) \int_{0}^{A} \lambda_{2}\left(a^{\prime}\right) p_{\infty}\left(a^{\prime}\right) v_{2}^{(n)}\left(a^{\prime}, t-a+\tau\right) d a^{\prime}\right)\right) \\
& +\eta e^{-\delta \tau} \lambda_{1}(\tau)\left(1-e^{\delta \tau} u_{1}^{(n)}(\tau, t-a+\tau)\right) \int_{0}^{A} \lambda_{2}\left(a^{\prime}\right) p_{\infty}\left(a^{\prime}\right) \\
& \left.\times\left(v_{2}^{(n)}\left(a^{\prime}, t-a+\tau\right)-v_{1}^{(n)}\left(a^{\prime}, t-a+\tau\right)\right) d a^{\prime}\right) d \tau \geq 0, \quad t \geq a .
\end{aligned}
$$


The selection for $\eta$ and $-\left(u_{2}^{(n)}(a, t)-u_{1}^{(n)}(a, t)\right) \geq-e^{\delta}\left(v_{2}^{(n)}(a, t)-v_{1}^{(n)}(a, t)\right)$ result in

$$
\begin{aligned}
& v_{2}^{(n+1)}(a, t)-v_{1}^{(n+1)}(a, t) \\
& =\exp \left(\frac{-t}{\eta}\right)\left(v_{2}^{(0)}(a, t)-v_{1}^{(0)}(a, t)\right)+\frac{1}{\eta} \int_{0}^{t} \exp \left(\frac{-(t-\tau)}{\eta}\right) \\
& \times\left(\left(v_{2}^{(n)}(a-t+\tau, \tau)-v_{1}^{(n)}(a-t+\tau, \tau)\right)(1-\eta(\gamma(a-t+\tau)+\delta))\right. \\
& -\eta e^{\delta(a-t+\tau)} \lambda_{1}(a-t+\tau)\left(u_{2}^{(n)}(a-t+\tau, \tau)\right. \\
& \left.-u_{1}^{(n)}(a-t+\tau, \tau)\right) \int_{0}^{A} \lambda_{2}\left(a^{\prime}\right) p_{\infty}\left(a^{\prime}\right) v_{2}^{(n)}\left(a^{\prime}, \tau\right) d a^{\prime} \\
& +\eta \lambda_{1}(a-t+\tau)\left(1-e^{\delta(a-t+\tau)} u_{1}^{(n)}(a-t+\tau, \tau)\right) \\
& \left.\times \int_{0}^{A} \lambda_{2}\left(a^{\prime}\right) p_{\infty}\left(a^{\prime}\right)\left(v_{2}^{(n)}\left(a^{\prime}, \tau\right)-v_{1}^{(n)}\left(a^{\prime}, \tau\right)\right) d a^{\prime}\right) d \tau \geq 0, \quad a \geq t
\end{aligned}
$$

and

$$
\begin{aligned}
& v_{2}^{(n+1)}(a, t)-v_{1}^{(n+1)}(a, t) \\
& =\frac{1}{\eta} \int_{0}^{a} \exp \left(\frac{-(a-\tau)}{\eta}\right)\left(\left(v_{2}^{(n)}(\tau, t-a+\tau)-v_{1}^{(n)}(\tau, t-a+\tau)\right)(1-\eta(\gamma(\tau)+\delta))\right. \\
& -\eta e^{\delta \tau} \lambda_{1}(\tau)\left(u_{2}^{(n)}(\tau, t-a+\tau)-u_{1}^{(n)}(\tau, t-a+\tau)\right) \int_{0}^{A} \lambda_{2}\left(a^{\prime}\right) p_{\infty}\left(a^{\prime}\right) v_{2}^{(n)}\left(a^{\prime}, t-a+\tau\right) d a^{\prime} \\
& +\eta \lambda_{1}(\tau)\left(1-e^{\delta \tau} u_{1}^{(n)}(\tau, t-a+\tau)\right) \int_{0}^{A} \lambda_{2}\left(a^{\prime}\right) p_{\infty}\left(a^{\prime}\right) \\
& \left.\times\left(v_{2}^{(n)}\left(a^{\prime}, t-a+\tau\right)-v_{1}^{(n)}\left(a^{\prime}, t-a+\tau\right)\right) d a^{\prime}\right) d \tau \geq 0, \quad t \geq a .
\end{aligned}
$$

From the above expressions, we deduce that

$$
\begin{array}{ll} 
& u_{2}^{(n+1)}(a, t)-u_{1}^{(n+1)}(a, t) \\
\leq & \exp \left(\delta a+\frac{-t}{\eta}\right)\left(v_{2}^{(0)}(a, t)-v_{1}^{(0)}(a, t)\right) \\
& +\frac{1}{\eta} \int_{0}^{t} \exp \left(\frac{-(t-\tau)}{\eta}\right)\left(\exp (\delta(a-t+\tau))\left(v_{2}^{(n)}(a-t+\tau, \tau)-v_{1}^{(n)}(a-t+\tau, \tau)\right)\right. \\
& \times\left(1-\eta\left(\gamma(a-t+\tau)+\delta+\lambda_{1}(a-t+\tau) \int_{0}^{A} \lambda_{2}\left(a^{\prime}\right) p_{\infty}\left(a^{\prime}\right) v_{2}^{(n)}\left(a^{\prime}, \tau\right) d a^{\prime}\right)\right) \\
& +\eta e^{-\delta(a-t+\tau)} \lambda_{1}(a-t+\tau)\left(1-e^{\delta(a-t+\tau)} u_{1}^{(n)}(a-t+\tau, \tau)\right) \\
& \left.\times \int_{0}^{A} \lambda_{2}\left(a^{\prime}\right) p_{\infty}\left(a^{\prime}\right)\left(v_{2}^{(n)}\left(a^{\prime}, \tau\right)-v_{1}^{(n)}\left(a^{\prime}, \tau\right)\right) d a^{\prime}\right) d \tau \\
& \leq e^{\delta a}\left(v_{2}^{(n+1)}(a, t)-v_{1}^{(n+1)}(a, t)\right), \quad a \geq t, \\
\text { thus } \left.\quad u_{2}^{(n+1)}(a, t)-u_{1}^{(n+1)}(a, t)\right) \leq e^{\delta a}\left(v_{2}^{(n+1)}(a, t)-v_{1}^{(n+1)}(a, t)\right), \quad t \geq a
\end{array}
$$


Therefore,

$$
u_{1}(a, t) \leq u_{2}(a, t) \quad \text { and } \quad v_{1}(a, t) \leq v_{2}(a, t)
$$

Hence given any constant $0 \leq \xi<1$, it is readily found that

$$
\xi F(u, v)(a, t) \leq F(\xi u, \xi v)(a, t), \quad \xi G(u, v)(a, t) \leq G(\xi u, \xi v)(a, t)
$$

From the construction of the iterative sequences in (24), we have that

$$
\xi u_{1}(a, t) \leq u_{\xi}(a, t), \quad \xi v_{1}(a, t) \leq v_{\xi}(a, t)
$$

and Theorem 3 is proved.

Theorem 4 Assume that $(A 1) \sim(A 6)$ hold. The positive equilibrium solution $u_{\infty}(a), v_{\infty}(a)$ of (22) is globally stable if $R_{0}>1$.

Proof. Using the method of characteristics, System (22) can be solved explicitly:

$$
\begin{aligned}
u(a, t) & =u_{0}(a-t) \exp \left(-\int_{0}^{t} \sigma_{1}(a-t+\tau, \tau) d \tau\right) \\
& +\int_{0}^{t} e^{-\delta(a-t+\tau)} \lambda_{1}(a-t+\tau) \omega(\tau) \exp \left(-\int_{\tau}^{t} \sigma_{1}(a-t+\theta, \theta) d \theta\right) d \tau, \quad a \geq t,(31 a) \\
u(a, t) & =\int_{0}^{a} e^{-\delta \tau} \lambda_{1}(\tau) \omega(t-a+\tau) \exp \left(-\int_{\tau}^{a} \sigma_{1}(\theta, t-a+\theta) d \theta\right) d \tau, \quad a \leq t, \\
v(a, t) & =v_{0}(a-t) \exp \left(-\int_{0}^{t} \sigma_{2}(a-t+\tau) d \tau\right) \\
& +\int_{0}^{t}\left(1-e^{\delta(a-t+\tau)} u(a-t+\tau, \tau)\right) \lambda_{1}(a-t+\tau) \omega(\tau) \\
& \times \exp \left(-\int_{\tau}^{t} \sigma_{2}(a-t+\theta) d \theta\right) d \tau, \quad a \geq t, \\
v(a, t) & =\int_{0}^{a}\left(1-e^{\delta \tau} u(\tau, t-a+\tau)\right) \lambda_{1}(\tau) \omega(t-a+\tau) \exp \left(-\int_{\tau}^{a} \sigma_{2}(\theta) d \theta\right) d \tau, a \leq t,(31 d)
\end{aligned}
$$

where,

$$
\begin{aligned}
& \omega(t)=\int_{0}^{A} \lambda_{2}(a) p_{\infty}(a) v(a, t) d a, \\
& \sigma_{1}(a, t)=\gamma(a)+\delta+\lambda_{1}(a) \omega(t), \\
& \sigma_{2}(a)=\gamma(a)+\delta .
\end{aligned}
$$

$\omega(t)>0$ for $0 \leq t \leq A$ holds from its definition. Combining (31b), (31d) and $e^{\delta a} u(a, t)<1(0<$ $a<A$ ) yields $u(a, A)>0$ and $v(a, A)>0$ for $0<a<A$. Continuing this process leads to 
$\omega(t)>0$ for $0 \leq t \leq 3 A$. Again from (31b) and (31d), we obtain

$$
\begin{array}{ll}
u(a, 2 A) \geq \int_{0}^{a} e^{-\delta \tau} \lambda_{1}(\tau) \omega_{0} \exp \left(-\int_{\tau}^{a} \sigma_{1}{ }^{*}(\theta, 2 A-a+\theta) d \theta\right) d \tau, & 0 \leq a \leq A,(32 \mathrm{a}) \\
v(a, 2 A) \geq \int_{0}^{a}\left(1-e^{\delta \tau} u(\tau, 2 A-a+\tau)\right) \lambda_{1}(\tau) \omega_{0} \exp \left(-\int_{\tau}^{a} \sigma_{2}(\theta) d \theta\right) d \tau, 0<a \leq A,(32 \mathrm{~b})
\end{array}
$$

where

$$
\begin{aligned}
& \omega_{0}=\min _{A \leq t \leq 2 A} \omega(t), \\
& \sigma_{1}^{*}(a, 2 A)=\gamma(a)+\delta+\lambda_{1}(a) \max _{A \leq t \leq 2 A} \int_{0}^{A} \lambda_{2}(\tau) p_{\infty}(\tau) v(\tau, t) d \tau .
\end{aligned}
$$

Moreover, from the expressions of $u_{\infty}(a)$ and $v_{\infty}(a)$ together (31) and (32), $\xi$ can be chosen such that

$$
\xi u_{\infty}(a) \leq u(a, 2 A) \leq e^{-\delta a}, \quad \xi v_{\infty}(a) \leq v(a, 2 A) \leq 1 .
$$

Let $u_{\xi}(a, t), v_{\xi}(a, t), u_{\delta}(a, t), v_{1}(a, t)$ be the solutions of (22) satisfying $u_{\xi}(a, 0)=\xi u_{\infty}(a)$, $v_{\xi}(a, 0)=\xi v_{\infty}(a), u_{\delta}(a, 0)=e^{-\delta a}, v_{1}(a, 0)=1$, respectively. By virtue of Theorem 3 , it is clear that

$$
\begin{aligned}
& u_{\xi}(a, t) \leq u(a, t+2 A) \leq u_{\delta}(a, t), \\
& v_{\xi}(a, t) \leq v(a, t+2 A) \leq v_{1}(a, t) .
\end{aligned}
$$

The fact that $u_{\infty}(a)$ and $v_{\infty}(a)$ are equilibrium solution implies that

$$
\begin{array}{ll}
\xi u_{\infty}(a) \leq u_{\xi}(a, t), & u_{\delta}(a, t) \leq e^{-\delta a} \\
\xi v_{\infty}(a) \leq v_{\xi}(a, t), & v_{1}(a, t) \leq 1
\end{array}
$$

which further imply that $u_{\xi}(a, t), v_{\xi}(a, t)$ are increasing, and conversely $u_{\delta}(a, t), v_{1}(a, t)$ are decreasing. Therefore, $\omega_{\xi}(t)=\int_{0}^{A} \lambda_{2}(a) p_{\infty}(a) v_{\xi}(a, t) d a$ is increasing and $\omega_{1}(t)=\int_{0}^{A} \lambda_{2}(a) p_{\infty}(a) v_{1}(a, t) d a$ is decreasing. Hence $\omega_{\xi}(t)$ and $\omega_{1}(t)$ must approach the same limit $\omega_{\infty}^{*}$, otherwise, the positive steady-state for (6) would not be unique. Hence the positive steady-state of (22) is globally asymptotically stable. This ends the proof of Theorem 4 .

From the assumptions and (2b), $G(a, t)$ can be expressed in terms of $u(a, t)$ and $v(a, t)$ as

$$
\begin{aligned}
G(a, t) & =\left(p_{\infty}(a)-j(a, t)\right) \int_{0}^{A} \lambda_{1}(a) \lambda_{2}\left(a^{\prime}\right) k\left(a^{\prime}, t\right) d a^{\prime} \\
& =\lambda_{1}(a) p_{\infty}(a)\left(1-e^{\delta a} u(a, t)\right) \int_{0}^{A} \lambda_{2}\left(a^{\prime}\right) p_{\infty}\left(a^{\prime}\right) v\left(a^{\prime}, t\right) d a^{\prime}
\end{aligned}
$$


Consequently, the solution of (2) is of the form

$$
i(a, c, t)=G(a-c, t-c) \pi\left(a^{\prime}, c\right), \quad t \geq A .
$$

where $G(a, t)$ is given above. Hence, the positive steady-state of (2) is globally asymptotically stable whenever $R_{0}>1$.

\section{Concluding remarks}

A general age-structured epidemic model is modified via the introduction of the infection age. The stability of steady-states and the uniqueness and existence of endemic steady-state for an SIS epidemic model are established . The basic reproductive number increases our understanding of the effect of infection age. Traditional single-age-structured model attempts to over estimate the disease severity. A two-age-structured SI epidemic model recently studied by Brauer[29] has also indicated this over estimation when the ratio of mean age at infection to the mean life span is very large.

It has been found that endemic equilibria are globally stable for epidemic models given by monotone ODE systems by Feng, Castillo-Chavez and Huang[30], Song and Castillo-Chavez [31], and Li [32]. Our work is an extension of this global dynamics to PDE system. We also expand the work of Thieme, Busenberg, Iannelli [9] to PDE systems with two-age structures. The approach of constructing iterate sequences to establish the global dynamics here may be useful in similar analysis of general epidemic models.

In the future, we intend to extend this approach to more realistic situations. For instance, we would like to apply it to fatal diseases and to situations that can handle general age distribution infection.

\section{Acknowledgments}

The valuable comments from Carlos Castillo-Chavez on our two manuscripts have improved this paper to a large extent. This work was partially supported by NSF and NSA grants to the Mathematical and Theoretical Biology Institute at Cornell University and the office of the Provost of Cornell University and by CNSF Grant 19971066 to Zhien Ma. 


\section{Appendix}

A rigorous proof of Theorem 1 is provided here.

Proof. Expression (6) helps us to equivalently show $G(a, t) \geq 0$. For the solution $i(a, c, t)$ of $(5)$ with the initial distribution satisfying the condition in Theorem 1, we define the age-structured distribution of the infectives by

$$
j(a, t)=\int_{0}^{a} i(a, c, t) d c=\int_{0}^{a} G(a-c, t-c) \frac{N(a)}{N(a-c)} d c=\int_{0}^{a} G(\tau, t-a+\tau) \frac{N(a)}{N(\tau)} d \tau,
$$

which satisfies

$$
\begin{aligned}
& \frac{\partial j}{\partial a}+\frac{\partial j}{\partial t}=-(\mu(a)+\gamma(a)) j(a, t)+G(a, t) \\
& j(0, t)=0 \\
& j(a, 0)=j_{0}(a)=\int_{0}^{a} i_{0}(a, c) d c .
\end{aligned}
$$

$u(a, t)=j(a, t) / p_{\infty}(a)$ is a normalization of $j(a, t)$, then

$$
\begin{aligned}
& \frac{\partial u}{\partial a}+\frac{\partial u}{\partial t}=-\gamma(a) u(a, t)+(1-u(a, t)) \int_{0}^{A} \int_{0}^{a^{\prime}} \lambda\left(a, a^{\prime}, c\right) i\left(a^{\prime}, c, t\right) d c d a^{\prime}, \\
& u(0, t)=0 \\
& u(a, 0)=u_{0}(a)=j_{0}(a) / p_{\infty}(a) .
\end{aligned}
$$

For any given $T(0<T<A)$ it follows from the given conditions of Theorem 1 that $i(a, c, t)$ is bounded for $0 \leq c \leq a \leq A, 0 \leq t \leq T$, and $0 \leq u_{0}(a) \leq 1$. Choose any positive constant $\eta$ and rewrite Equation (34a) as

$$
\begin{aligned}
\frac{\partial u}{\partial a}+\frac{\partial u}{\partial t}= & -\frac{u(a, t)}{\eta}+\frac{u(a, t)}{\eta}-\gamma(a) u(a, t) \\
& +(1-u(a, t)) \int_{0}^{A} \int_{0}^{a^{\prime}} \lambda\left(a, a^{\prime}, c\right) i\left(a^{\prime}, c, t\right) d c d a^{\prime}
\end{aligned}
$$


from which an equivalent integral equations is deduced

$$
\begin{array}{rlr}
u(a, t) & =\exp \left(\frac{-t}{\eta}\right) u_{0}(a-t)+\frac{1}{\eta} \int_{0}^{t} \exp \left(\frac{-(t-\tau)}{\eta}\right)(u(a-t+\tau, \tau) & \\
& +\eta(-\gamma(a-t+\tau) u(a-t+\tau, \tau)+(1-u(a-t+\tau, \tau)) & \\
& \left.\left.\times \int_{0}^{A} \int_{0}^{a^{\prime}} \lambda\left(a-t+\tau, a^{\prime}, c\right) i\left(a^{\prime}, c, \tau\right) d c d a^{\prime}\right)\right) d \tau, & \\
u(a, t) & =\frac{1}{\eta} \int_{0}^{a} \exp \left(\frac{-(a-\tau)}{\eta}\right)(u(\tau, t-a+\tau) \\
& +\eta(-\gamma(\tau) u(\tau, t-a+\tau)+(1-u(\tau, t-a+\tau)) \\
& \left.\left.\times \int_{0}^{A} \int_{0}^{a^{\prime}} \lambda\left(\tau, a^{\prime}, c\right) i\left(a^{\prime}, c, t-a^{\prime}+\tau\right) d c d a^{\prime}\right)\right) d \tau, \quad a<t,
\end{array}
$$

where $\eta$ is a positive constant, such that $\eta\left(\gamma(a)+\int_{0}^{A} \int_{0}^{a^{\prime}} \lambda\left(a, a^{\prime}, c\right)\left|i\left(a^{\prime}, c, t\right)\right| d c d a^{\prime}\right)<1$. We apply the routine iterative procedure

$$
\begin{array}{rlr}
u^{(0)}(a, t)= & u_{0}(a) \\
u^{(n+1)}(a, t) & =\exp \left(\frac{-t}{\eta}\right) u_{0}(a-t)+\frac{1}{\eta} \int_{0}^{t} \exp \left(\frac{-(t-\tau)}{\eta}\right)\left(u^{(n)}(a-t+\tau, \tau)\right. \\
& +\eta\left(-\gamma(a-t+\tau) u^{(n)}(a-t+\tau, \tau)+\left(1-u^{(n)}(a-t+\tau, \tau)\right)\right. \\
& \left.\left.\times \int_{0}^{A} \int_{0}^{a^{\prime}} \lambda\left(a-t+\tau, a^{\prime}, c\right) i\left(a^{\prime}, c, \tau\right) d c d a^{\prime}\right)\right) d \tau, \quad a \geq t \\
u^{(n+1)}(a, t) & =\frac{1}{\eta} \int_{0}^{a} \exp \left(\frac{-(a-\tau)}{\eta}\right)\left(u^{(n)}(\tau, t-a+\tau)\right. & \\
& +\eta\left(-\gamma(\tau) u^{(n)}(\tau, t-a+\tau)+\left(1-u^{(n)}(\tau, t-a+\tau)\right)\right. & \\
& \left.\left.\times \int_{0}^{A} \int_{0}^{a^{\prime}} \lambda\left(\tau, a^{\prime}, c\right) i\left(a^{\prime}, c, t-a+\tau\right) d c d a^{\prime}\right)\right) d \tau, \quad a<t .
\end{array}
$$

One can easily show that the iterative sequence $u^{(n)}(a, t)$ converges uniformly to the solution of the integral equation (35). 
If $0 \leq u^{(n)}(a, t) \leq 1$, then we can obtain from (36b) and (36c) that

$$
\begin{aligned}
u^{(n+1)}(a, t) & \leq \exp \left(\frac{-t}{\eta}\right) u_{0}(a-t)+\frac{1}{\eta} \int_{0}^{t} \exp \left(\frac{-(t-\tau)}{\eta}\right)\left(u^{(n)}(a-t+\tau, \tau)\right. \\
& +\eta\left(\gamma(a-t+\tau)\left(1-u^{(n)}(a-t+\tau, \tau)\right)+\left(1-u^{(n)}(a-t+\tau, \tau)\right)\right. \\
& \left.\left.\times \int_{0}^{A} \int_{0}^{a^{\prime}} \lambda\left(a-t+\tau, a^{\prime}, c\right)\left|i\left(a^{\prime}, c, \tau\right)\right| d c d a^{\prime}\right)\right) d \tau \\
& \leq \exp \left(\frac{-t}{\eta}\right) u_{0}(a-t)+\frac{1}{\eta} \int_{0}^{t} \exp \left(\frac{-(t-\tau)}{\eta}\right)\left(u^{(n)}(a-t+\tau, \tau)\right. \\
& \left.+\left(1-u^{(n)}(a-t+\tau, \tau)\right) \eta\left(\gamma(a-t+\tau)+\int_{0}^{A} \int_{0}^{a^{\prime}} \lambda\left(a-t+\tau, a^{\prime}, c\right)\left|i\left(a^{\prime}, c, \tau\right)\right| d c d a^{\prime}\right)\right) d \tau \\
& \leq \exp \left(\frac{-t}{\eta}\right)+\frac{1}{\eta} \int_{0}^{t} \exp \left(\frac{-(t-\tau)}{\eta}\right)\left(u^{(n)}(a-t+\tau, \tau)+\left(1-u^{(n)}(a-t+\tau, \tau)\right)\right) d \tau \\
& \leq \exp \left(\frac{-t}{\eta}\right)+\frac{1}{\eta} \int_{0}^{t} \exp \left(\frac{-(t-\tau)}{\eta}\right) d \tau \leq 1, \quad a \geq t, \quad(37 \mathrm{a}) \\
u^{(n+1)}(a, t) & \leq \frac{1}{\eta} \int_{0}^{a} \exp \left(\frac{-(a-\tau)}{\eta}\right) d \tau \leq 1, \quad a>t .
\end{aligned}
$$

where Inequality (37b) is deduced by the same process as does for (37a). Hence, induction implies that $u^{(n)}(a, t) \leq 1$ for all integer $n$, by which we arrive at

$$
\begin{aligned}
& \lim _{n \rightarrow \infty} u^{(n)}(a, t)=u(a, t) \leq 1, \\
& j(a, t) \leq p_{\infty}(a), \quad 0 \leq a \leq A, \quad 0 \leq t \leq T .
\end{aligned}
$$

Recalling Equation (6) and (7), we find

$$
\begin{aligned}
& G(a, t)=f(a, t)+h(a, t) \int_{0}^{t} \int_{c}^{A} \lambda\left(a, a^{\prime}, c\right) G\left(a^{\prime}-c, t-c\right) \pi\left(a^{\prime}, c\right) d a^{\prime} d c, \\
& \text { where } f(a, t)=h(a, t) \int_{t}^{A} \int_{c}^{A} \lambda\left(a, a^{\prime}, c\right) i_{0}\left(a^{\prime}-t, c-t\right) \pi\left(a^{\prime}, t\right) d a^{\prime} d c \\
& h(a, t)=p_{\infty}(a)-j(a, t), \quad 0 \leq a \leq A, \quad 0 \leq t \leq A .
\end{aligned}
$$

$f(a, t)$ and $h(a, t)$ are nonnegative continuous and bounded on $D=[0, A] \times[0, A]$. In order to prove the nonnegativity of $G(a, t)$ we built a new sequence by iteration as follows

$$
\begin{array}{rlrl}
G^{(0)}(a, t) & =i_{0}(a, t), & 0 & \leq t \leq a \leq A, \\
G^{(0)}(a, t) & =i_{0}(a, a), & 0 & \leq a \leq t \leq A, \\
G^{(n)}(a, t) & =f(a, t)+h(a, t) \int_{0}^{t} \int_{c}^{A} \lambda\left(a, a^{\prime}, c\right) G^{(n-1)}\left(a^{\prime}-c, t-c\right) \pi\left(a^{\prime}, c\right) d a^{\prime} d c \\
& (a, t) \in D, \quad n=1,2, \ldots .
\end{array}
$$


Owing to the fact that $G^{(0)}(a, t)$ is a nonnegative and continuous on $D, G^{(n)}(a, t)$ are also nonnegative and continuous on $D$ for all $n$. Denoting $h^{*}=\max _{(a, t) \in D} h(a, t)$, we can estimate the norm between two consecutive terms in the sequence, resulting in

$$
\left\|G^{(n+1)}(a, t)-G^{(n)}(a, t)\right\| \leq h^{*} A \lambda^{*}\left\|G^{(n)}(a, t)-G^{(n-1)}(a, t)\right\| t .
$$

Inequality (41) together with the induction imply that

$$
\left\|G^{(n+1)}(a, t)-G^{(n)}(a, t)\right\| \leq\left(h^{*} A \lambda^{*}\right)^{n}\left\|G^{(1)}(a, t)-G^{(0)}(a, t)\right\| t^{n} / n !
$$

from which it follows that $G^{(n)}(a, t)$ converges uniformly on $D$. And thus $G(a, t)=\lim _{n \rightarrow \infty} G^{(n)}(a, t) \geq$ 0 holds for $0 \leq t \leq A$. This is also true for all $t$ by repeating the process. We finish the proof of Theorem 1.

\section{References}

1. W.O. Kermack and A.G. Mckendrick, Contributions to the theory of epidemics, Proc. Roy. Soc., A, 115, 700-721(1927).

2. A.G. Mckendrick, Applications of mathematics to medical problems. Proc. Edinburgh Math. Soc., 44, 98-130(1926).

3. F. Hoppensteadt, An age dependent epidemic model, J. Franklin Inst., 297, 325-333(1974).

4. F. Hoppensteadt, Mathematical theories of populations: demographics, genetics and epidemics, SIAM, Philadelphia(1975).

5. P. Waltman, Deterministic threshold models in the theory of epidemics, Lecture Notes in Biomathematics 1, Springer-Verlag, Berlin, New York(1974).

6. E. Vynnycky and P.E. Fine, The natural history of tuberculosis: the implications of agedependent risks of disease and the role of reinfection. Epidemiol. Infect., 19,183-201(1997).

7. C. Castillo-Chavez and Z. Feng, Global stability of an age-structure model for TB and its application to optimal vaccination strategies, Math. Bioscie., 151, 135-154(1998).

8. S. Busenberg, K. Cooke, M. Iannelli, Endemic thresholds and stability in a class of agestructured epidemics, SIAM J. Appl. Math., 48(6), 1379-1395(1988). 
9. S. Busenberg, M. Iannelli, H.R. Thieme, Global behavior of an age-structured epidemic model, SIAM J. Math. Anal., 22(4), 1065-1080(1991).

10. M. Iannelli, F.A. Milner, A. Pugliese, Analytical and numerical results for the age-structured S-I-S epidemic model with mixed inter-intracohort transmission, SIAM J. Math. Anal., 23(3), 662-688(1992).

11. Y. Cha, M. Iannelli, F.A. Milner, Existence and uniqueness of endemic states for the age-structured S-I-R epidemic model, Math. Bioscie., 150, 177-190(1998).

12. H.W. Hethcote, A thousand and one epidemic models, in: Frontiers in Mathematical Biology, Simon A. Levin eds, Lecture Notes in Biomathematics 100, Springer-Verlag, Berlin, Heidelberg, 504-515(1994).

13. V. Capasso, Mathematical structures of epidemic systems, Lecture Notes in Biomathematics 97, Springer-Verlag, Berlin, Haidelberg(1993).

14. M. Iannelli, Mathematical theory of age-structured population dynamics, Giardini Editori, E Stampatori, in Pisa, 1995.

15. C. Castillo-Chavez, H.W. Hethcote, V. Andreasen, S.A. Levin, and W.M. Liu, Epidemiological models with age-structure, proportionate mixing, and cross-immunity, J. Math. Biol., 27, 233-258(1989).

16. H.W. Hethcote, and J.A. Yorke, Gonorrhea, transmission, dynamics, and control, Lecture Notes in Biomathematics, 56, Springer-Verlag, Berlin, New York(1984).

17. K. Dietz, D. Schenzle, Proportionate mixing models for age-dependent infection transmission, J. of Math. Biol., 22, 117-120(1985).

18. H.R. Thieme, C. Castillo-Chavez, How may infection-age-dependent infectivity affect the dynamics of HIV/AIDs? SIAM J. Appl. Math., 53(5), 1447-1479(1993).

19. O. Diekmann, J.A.P. Heesterbeek, and J.A.J. Mets, On the definition and the computation of basic reproductive ratio $R_{0}$ in models for infectious diseases in heterogeneous population, J. Math. Biol. 28, 365-382(1990) 
20. S. Busenberg and C. Castillo-Chavez, A general solution of the problem of mixing of subpopulations and its application to risk- and age-structured epidemic models for the spread of AIDS, IMA J. Math. Appl. Med. Biol., 8, 1-29(1990)

21. Song Jian, Yu Jingyuan, Population system control, Springer-Verlag, Berlin(1987).

22. J.M. Cushing, An introduction to structured population dynamics, Society for Industrial and Applied Mathematics, Philadelphia(1998).

23. M.E. Gurtin and R.C. MacCamy, Nonlinear age-dependent population dynamics, Archive for Rational Mechanics and Analysis, 54(3), 281-300(1974).

24. G.F. Webb, Theory of nonlinear age-dependent population dynamics, Marcel Dekker, New York(1985).

25. J. Pruss, Stability analysis for equilibria in an age-specific population dynamics, Nonlinear Analysis TMA, 7(12), 1291-1313(1983).

26. Y. Zhou, The continuous solutions and average net reproductive number of an age structured population model, in: Advanced topics in biomathematics, Lansun Chen, Shigui Ruan, Jun Zhu, eds., World Scientific, Singapore, 315-320(1998).

27. Y. Zhou and Z. Ma, The global stability of the positive equilibrium of a nonlinear agestructured population model, J. of Xi'an Jiaotong University, 33(10), 87-90, 1999.

28. S.L. Tucker, S.O. Zimmerman, A nonlinear model of population dynamics containing an arbitrary number of continuous structure variables, SIAM J. Appl. Math., 48(3), 549591(1988).

29. F. Brauer, Infectious diseases models with chronological age structure and epidemiological age structure, (this volume).

30. Z. Feng, C. Castillo-Chavez, W. Huang, On the role of variable latent period in mathematical models for tuberculosis, Journal of Dynamics and Different Equations (in process), Vol. 13, (2001) 
31. B. Song and C. Castillo-Chavez and J.P. Aparicio, Global Dynamics Analysis of TB Models with Density-dependent Demography, (this volume)

32. M.Y. Li, J. R. Graef, L.C. Wang and J. Karsai, Global dynamics of an SEIR model with varying total population size, Math. Biosci, 160, 191-213 (1999) 